\title{
Reengineering a transmembrane protein to treat muscular dystrophy using exon skipping
}

\author{
Quan Q. Gao, ${ }^{1}$ Eugene Wyatt, ${ }^{2}$ Jeff A. Goldstein, ${ }^{3}$ Peter LoPresti, ${ }^{3}$ Lisa M. Castillo, ${ }^{2}$ Alec Gazda, ${ }^{3}$ Natalie Petrossian, ${ }^{3}$ \\ Judy U. Earley, ${ }^{2}$ Michele Hadhazy, ${ }^{2}$ David Y. Barefield, ${ }^{2}$ Alexis R. Demonbreun, ${ }^{2}$ Carsten Bönnemann, ${ }^{4}$ \\ Matthew Wolf, ${ }^{5}$ and Elizabeth M. McNally ${ }^{2}$ \\ 'Committee on Development, Regeneration, and Stem Cell Biology, The University of Chicago, Chicago, Illinois, USA. 'Center for Genetic Medicine, Northwestern University, Evanston, Illinois, USA. \\ ${ }^{3}$ Department of Pathology, The University of Chicago, Chicago, Illinois, USA. ${ }^{4}$ National Institute of Neurological Diseases and Stroke, NIH, Bethesda, Maryland, USA. ${ }^{5}$ Department of Medicine, \\ Duke University, Durham, North Carolina, USA.
}

\begin{abstract}
Exon skipping uses antisense oligonucleotides as a treatment for genetic diseases. The antisense oligonucleotides used for exon skipping are designed to bypass premature stop codons in the target RNA and restore reading frame disruption. Exon skipping is currently being tested in humans with dystrophin gene mutations who have Duchenne muscular dystrophy. For Duchenne muscular dystrophy, the rationale for exon skipping derived from observations in patients with naturally occurring dystrophin gene mutations that generated internally deleted but partially functional dystrophin proteins. We have now expanded the potential for exon skipping by testing whether an internal, in-frame truncation of a transmembrane protein $\gamma$-sarcoglycan is functional. We generated an internally truncated $\gamma$-sarcoglycan protein that we have termed Mini-Gamma by deleting a large portion of the extracellular domain. Mini-Gamma provided functional and pathological benefits to correct the loss of $\gamma$-sarcoglycan in a Drosophila model, in heterologous cell expression studies, and in transgenic mice lacking $\gamma$-sarcoglycan. We generated a cellular model of human muscle disease and showed that multiple exon skipping could be induced in RNA that encodes a mutant human $\gamma$-sarcoglycan. Since Mini-Gamma represents removal of 4 of the 7 coding exons in $\gamma$-sarcoglycan, this approach provides a viable strategy to treat the majority of patients with $\gamma$-sarcoglycan gene mutations.
\end{abstract}

\section{Introduction}

Mutations that disrupt the dystrophin glycoprotein complex (DGC) cause muscular dystrophy (1-3). Dystrophin and its associated proteins localize to the muscle plasma membrane, acting as a linker between the intracellular cytoskeleton and the extracellular matrix $(4,5)$. Large deletions in the dystrophin gene account for Duchenne muscular dystrophy (DMD). Mutations that result in internal deletions and maintain the reading frame of dystrophin cause the milder Becker muscular dystrophy (BMD). These observations in BMD provided the basis for developing antisense oligonucleotide $(\mathrm{AON})$ therapies for the treatment of DMD with the goal of inducing exon-skipping events to restore reading frame. DMD exon skipping, by design, generates an internally truncated and partially functional protein. Clinically, exon skipping is expected to convert severe DMD patients into milder BMD patients, resulting in prolonged ambulation and better maintenance of muscle strength. Clinical trials that test exon skipping in DMD are advancing (6-10). Dys-

Note regarding evaluation of this manuscript: Manuscripts authored by scientists associated with Duke University, The University of North Carolina at Chapel Hill, Duke-NUS, and the Sanford-Burnham Medical Research Institute are handled not by members of the editorial board but rather by the science editors, who consult with selected external editors and reviewers.

Conflict of interest: Patents have been filed by the University of Chicago and Northwestern University related to this work.

Submitted: May 13, 2015; Accepted: September 3, 2015.

Reference information: J Clin Invest. 2015;125(11):4186-4195. doi:10.1172/JCI82768. trophin, with its highly repetitive internal structure composed of 24 spectrin repeats, is ideal for exon skipping. Moreover, since the gene encoding dystrophin is located on the X-chromosome, AONs need only target the mRNA produced from 1 allele.

The sarcoglycan subcomplex within the DGC is composed of 4 single-pass transmembrane subunits: $\alpha-, \beta-, \gamma-$, and $\delta$-sarcoglycan $(11,12)$. Recessive loss-of-function mutations in genes encoding $\alpha^{-}, \beta-, \gamma^{-}$, and $\delta$-sarcoglycan cause the limb girdle muscular dystrophies (LGMD) type 2D, 2E, 2C, and 2F, respectively (13-16). Here, we examined the plausibility of applying an exon-skipping strategy to treat LGMD 2C patients with mutations in $S G C G$, the gene encoding $\gamma$-sarcoglycan. The most common mutation in LGMD 2C patients is a deletion of a thymine from a string of 5 thymines at nucleotide bases $521-$ 525 in exon 6 of the $\gamma$-sarcoglycan gene, referred to as $521-\Delta \mathrm{T}$ (15). This mutation shifts the reading frame and results in the absence of $\gamma$-sarcoglycan protein and secondary reduction of $\beta$ - and $\delta$-sarcoglycans (15). To skip this mutation and restore the reading frame requires the skipping of exons $4,5,6$, and 7 . This internally truncated protein, which we refer to as MiniGamma, retains the intracellular, transmembrane, and extreme carboxy-terminus. To assess Mini-Gamma's capacity to substitute for full-length $\gamma$-sarcoglycan, we generated both transgenic flies and mice expressing Mini-Gamma, finding functional and molecular evidence for rescue of $\gamma$-sarcoglycan deficiency. We also provide proof-of-principle evidence that exon skipping can be induced in SGCG mutant human cells. 
A

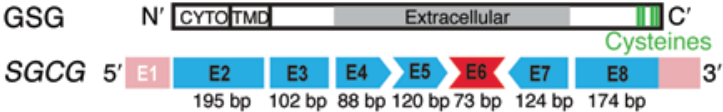

\begin{tabular}{l|l|l|l|l|l} 
Mini-Gamma 5' E1 & E2 & E3 & E8 & $3^{\prime}$
\end{tabular}

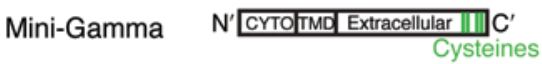

$\mathbf{B}$
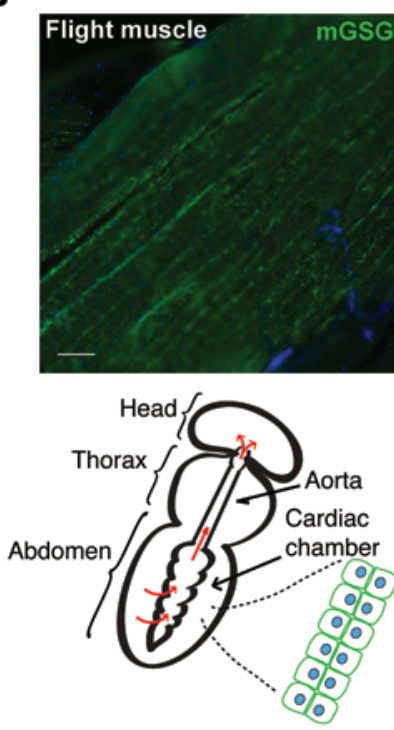





C
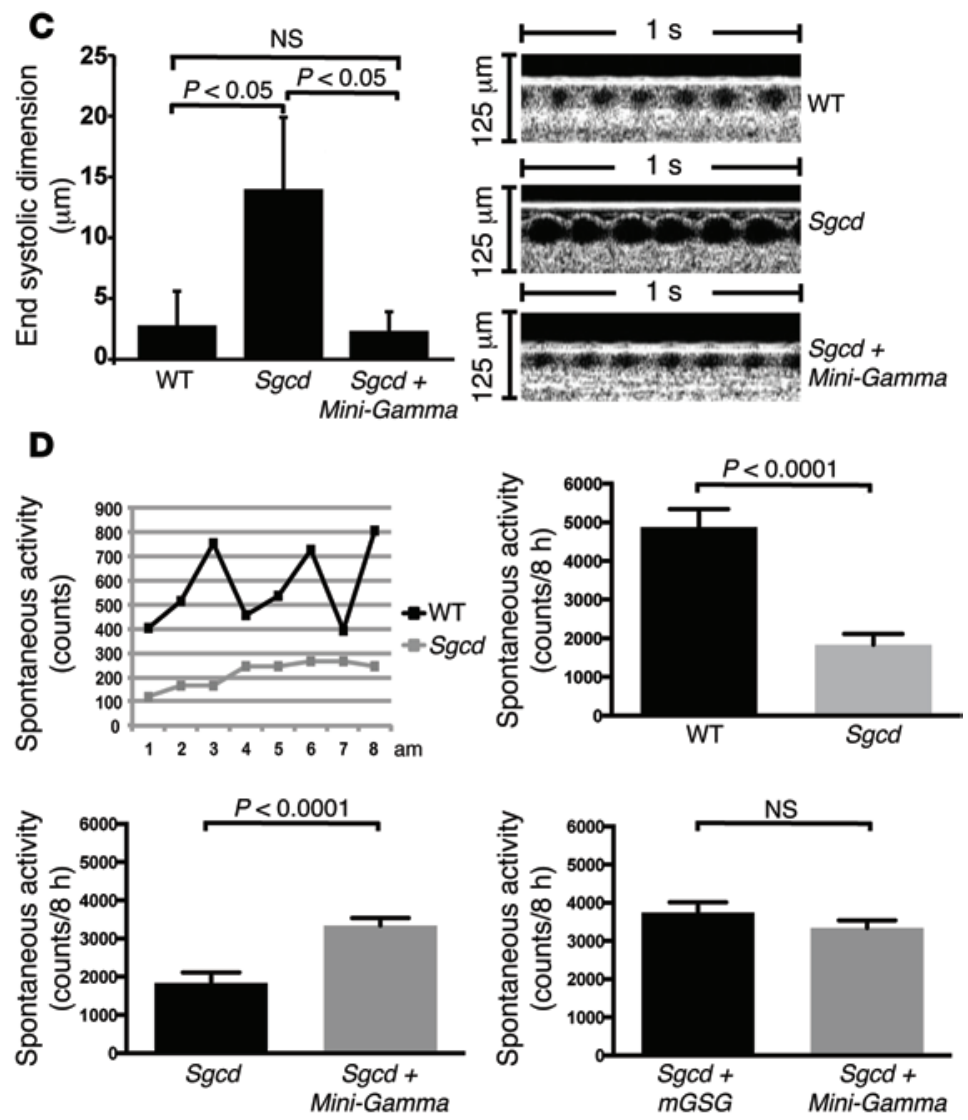

Figure 1. Mini-Gamma rescues Drosophila muscular dystrophy. (A) $\gamma$-Sarcoglycan (GSG) is a type II transmembrane protein with a cytoplasmic amino-terminus and an extracellular carboxy-terminus. The SGCG gene encoding $\gamma$-sarcoglycan is composed of 8 exons, and the most common mutation falls within exon 6 and disrupts the reading frame (15). To restore the reading frame, skipping exon 4-7 is required. This approach removes a portion of the extracellular domain, producing an internally truncated protein, referred to as Mini-Gamma. (B) The GAL4/UAS system was used to express fulllength mGSG and Mini-Gamma as transgenes in $S g c d^{840}$ flies, a sarcoglycan-deficient model of muscular dystrophy. Mini-Gamma protein localized to the plasma membrane in Sgcd ${ }^{840}$ fly skeletal muscle (Mef2-Gal4, UAS-Mini-Gamma), similar to full-length mGSC (Mhc-Gal4, UAS-mGSG). In fly heart tube, Mini-Gamma also showed a plasma membrane staining (Tin_C-Gal4, UAS-Mini-Gamma). Scale bars: $20 \mu \mathrm{m}$. (C) OCT was used to measure fly heart function (24). $S g c d^{840}$ flies had dilated heart tubes with increased ESD compared with WT flies. Expression of Mini-Camma in the $S g c d^{840}$ heart tube restored ESD to WT level (Mef-Gal4, UAS-Mini-Gamma) ( $n=10 \sim 12$ flies per genotype). (D) The MB5 monitor was used to record fly spontaneous activity. Nocturnal activity is shown (12 am-8 am). Expression of Mini-Gamma improved nocturnal activity of Sgcd ${ }^{840}$. The degree of rescue was similar between full-length mGSG and Mini-Gamma ( $n=20 \sim 35$ flies per genotype). Student's $t$ test was used to compare results between 2 groups.

\section{Results}

Expression of murine Mini-Gamma rescues a Drosophila model of muscular dystrophy. $\gamma$-Sarcoglycan is a type II transmembrane protein with a short intracellular domain, a single transmembrane pass, and a larger carboxy-terminal extracellular domain. A transgene was engineered to express an internally truncated $\gamma$-sarcoglycan. The internally truncated protein, referred to as Mini-Gamma, reflects the deletion of the protein regions encoded by exons 4,5 , 6 , and 7 (Figure 1A). To test the functionality of Mini-Gamma, the GAL4/UAS system was used to express murine Mini-Gamma in a previously established Drosophila model of muscular dystrophy $(17,18)$. Drosophila has a single $\gamma / \delta$-sarcoglycan ortholog that is equally related to mammalian $\gamma$ - and $\delta$-sarcoglycan (35\% identical, $56 \%$ similar to each). $S g c d^{840}$ flies have a large deletion of the Drosophila $S g c d$ gene, and $S g c d^{840}$ flies develop impaired motility and dilated heart tubes in adulthood (18). The sarcoglycan complex is localized at the muscle membrane, and loss-of-function mutations in mice and humans result in the absence of plasma membrane-associated staining (19-23). Full-length murine $\gamma$-sarcoglycan (mGSG) localized to the sarcolemma when expressed in $S g c d^{840}$ muscle (Figure 1B), indicating that the mGSG normally translocates in Drosophila muscle. Expression of murine MiniGamma showed the same distinct plasma membrane localization when expressed in $S g c d^{840}$ flies (Figure 1B). Expression of MiniGamma in $\mathrm{Sgcd}^{840}$ hearts also showed plasma membrane-associated staining in the thin-walled heart tube structure (Figure 1B, lower panels). Expression of Mini-Gamma in WT flies showed less distinct membrane localization and more accumulation of MiniGamma in the ER-like structure surrounding nuclei (Supplemental Figure 1; supplemental material available online with this article; doi:10.1172/JCI82768DS1). This decrement in Mini-Gamma membrane staining is consistent with competition between MiniGamma and the endogenous fly $S g c d$-encoded protein.

To measure Drosophila heart function, optical coherence tomography (OCT) was used to measure heart tube dimension during both contraction and relaxation (24). $S g c d^{840}$ flies had dilated 
A Transfect with

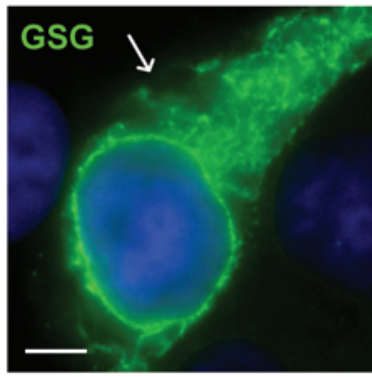

Transfect with

Mini-Gamma

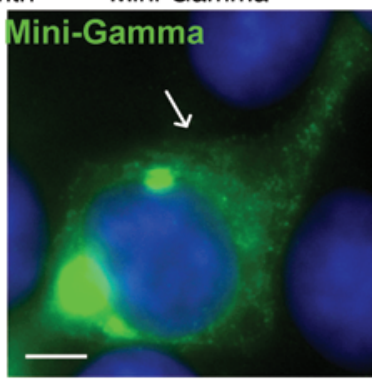

+BSG+DSG

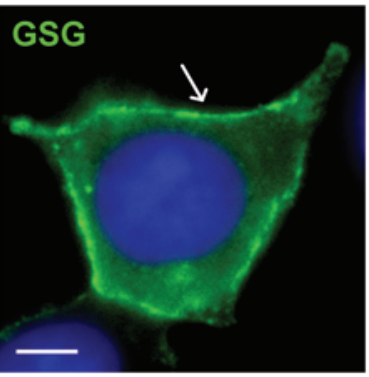

+BSG+DSG



B
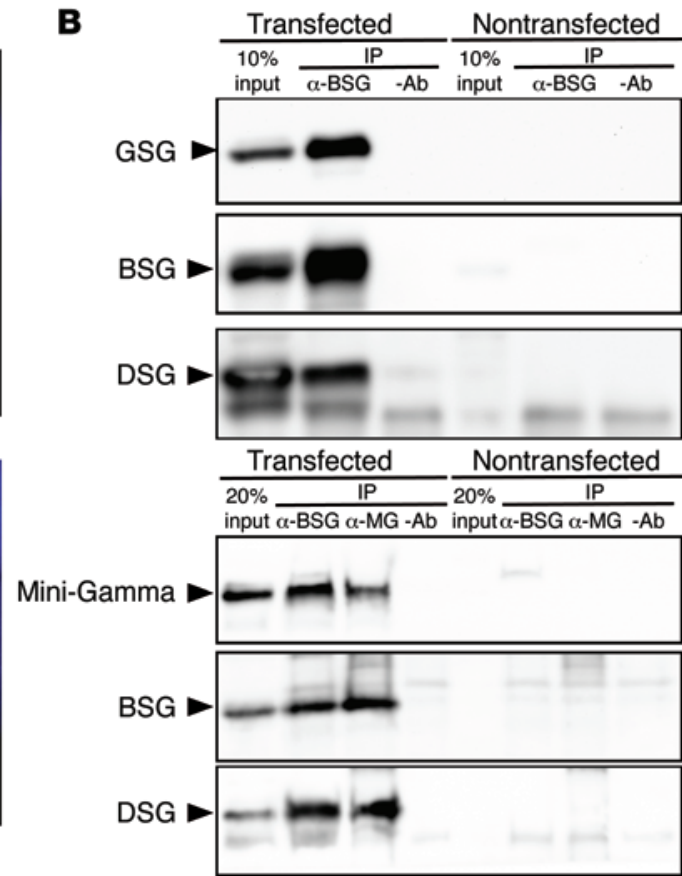

Figure 2. Mini-Gamma interacts with $\boldsymbol{\beta}$ - and $\boldsymbol{\delta}$-sarcoglycans. Plasmids encoding mammalian sarcoglycans were expressed in HEK293T cells. (A) Expression of either Mini-Gamma or full-length $\gamma$-sarcoglycan (GSC) alone resulted in cytoplasmic and perinuclear accumulation. This observation is consistent with previous reports that association with the $\beta / \delta$-sarcoglycan core is required for membrane targeting (26). Arrows in left panels indicate little to no plasma membrane trafficking. Coexpression of full-length $\gamma$-sarcoglycan with $\beta$ - and $\delta$-sarcoglycan (BSC and DSG) resulted in plasma membrane translocation of $\gamma$-sarcoglycan (arrow in top right panel). Similarly, expression of Mini-Gamma with $\beta$ - and $\delta$-sarcoglycan resulted in plasma membrane translocation of Mini-Gamma (arrow in bottom right panel). Scale bars: $5 \mu \mathrm{m}$. (B) Co-IP was performed to examine sarcoglycan-complex formation from HEK293T heterologous cell-expression experiments. After IP with an anti- $\beta$-sarcoglycan antibody, a complex containing $\beta-,-\delta$-, and $\gamma$-sarcoglycan was detected in $\beta / \delta / \gamma$ coexpressing cells (upper panels). Likewise, IP with the same anti- $\beta$-sarcoglycan antibody demonstrated an interaction among $\beta$ - and $\delta$-sarcoglycan and Mini-Gamma (lower panels). IP for Mini-Gamma using an antibody against the Xpress tag also detected $\beta$ - and $\delta$-sarcoglycan. MG, Mini-Gamma.

heart tubes with significantly increased end systolic dimension (ESD) compared with WT (Figure 1C). Expression of Mini-Gamma in the heart tube was sufficient to restore ESD to WT dimensions (Figure 1C). A representative OCT tracing demonstrates the dilated nature in $\mathrm{Sgcd}^{840}$ heart tubes and rescue of this phenotype by transgenic expression of Mini-Gamma (Figure 1C). $S g c d^{840}$ flies display locomotive defects as a result of skeletal muscle degeneration (18). A Drosophila activity monitor was used to record fly spontaneous activity over 24-48 hours (Supplemental Figure 2), and nocturnal activity was compared between WT and $S g c d^{840}$ flies. $S g c d^{840}$ flies had reduced activity (Figure 1D), consistent with other assays of mobility (18). Expression of Mini-Gamma in Sgcd ${ }^{840}$ flies significantly improved the activity of $S g c d^{840}$ flies (Figure 1D) but did not fully restore $S g c d^{840}$ fly mobility to WT activity level. However, we noted that expression of $\mathrm{mGSG}$ resulted in similar level of rescue of activity as Mini-Gamma (Figure 1D, lower right, and Supplemental Figure 3) suggesting that at least part of the failure to fully restore activity derives from the differences between Drosophila and mammalian sarcoglycans.

Mini-Gamma interacts with other sarcoglycans. Murine sarcoglycan proteins were transiently expressed in human embryonic kidney (HEK 293T) cells to examine their intracellular localization. It was previously shown that $\beta$ - and $\delta$-sarcoglycan form a core subunit, followed by the addition of $\gamma$-sarcoglycan to the complex (21, $25,26)$. Expression of the individual sarcoglycan subunits $-\beta-, \gamma^{-}$, or $\delta$-sarcoglycan or Mini-Gamma - produced little accumulation of immunoreactivity at the plasma membrane (Figure 2A and data not shown), consistent with prior reports of interdependency for normal intracellular trafficking (26). Coexpression of $\beta$ - and $\delta$-sarcoglycan, along with $\gamma$-sarcoglycan, resulted in plasma membrane enrichment of $\gamma$-sarcoglycan (Figure 2A, upper panel right). Similarly, expression of $\beta$ - and $\delta$-sarcoglycan and Mini-Gamma also resulted in plasma membrane-associated Mini-Gamma staining (Figure 2A, lower panel right). $\gamma$-Sarcoglycan and Mini-Gamma translocated similarly to the membrane when each was expressed in the presence of $\beta$ - and $\delta$-sarcoglycan (Supplemental Figure 4). IP of expressed sarcoglycan subunits using an anti- $\beta$-sarcoglycan antibody confirmed the presence of complexes containing $\beta-, \gamma^{-}$, and $\delta$-sarcoglycan (Figure $2 \mathrm{~B}$, top panels). Likewise, IP with an anti- $\beta$-sarcoglycan antibody demonstrated an interaction among $\beta$-sarcoglycan, $\delta$-sarcoglycan, and Mini-Gamma (Figure 2B, bottom panels). IP for Mini-Gamma also detected $\beta$ - and $\delta$-sarcoglycan (Figure 2B, bottom panels). These data demonstrate that Mini-Gamma formed a complex with $\beta$ - and $\delta$-sarcoglycan like full-length $\gamma$-sarcoglycan.

Mini-Gamma is incorporated into the sarcoglycan complex in vivo. To test the function of Mini-Gamma in vivo, transgenic mice expressing Mini-Gamma under the control of the desmin promoter were generated. The desmin promoter is active in both heart and skeletal muscle (27). Two lines were characterized. Line Tg50 demonstrated higher-level expression, with Mini-Gamma expressed at 3 times the level of endogenous $\gamma$-sarcoglycan pro- 

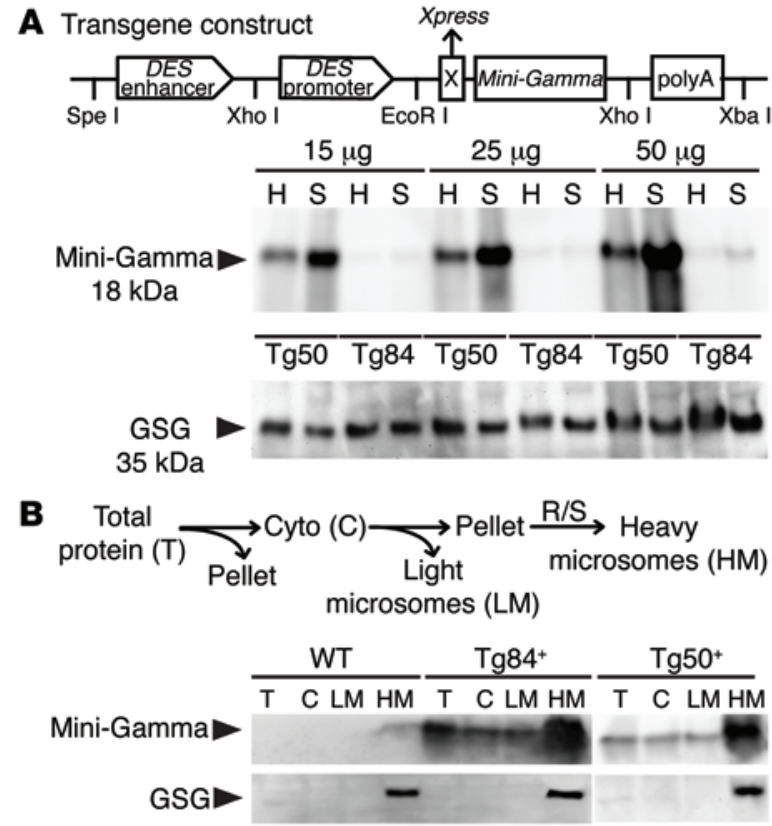

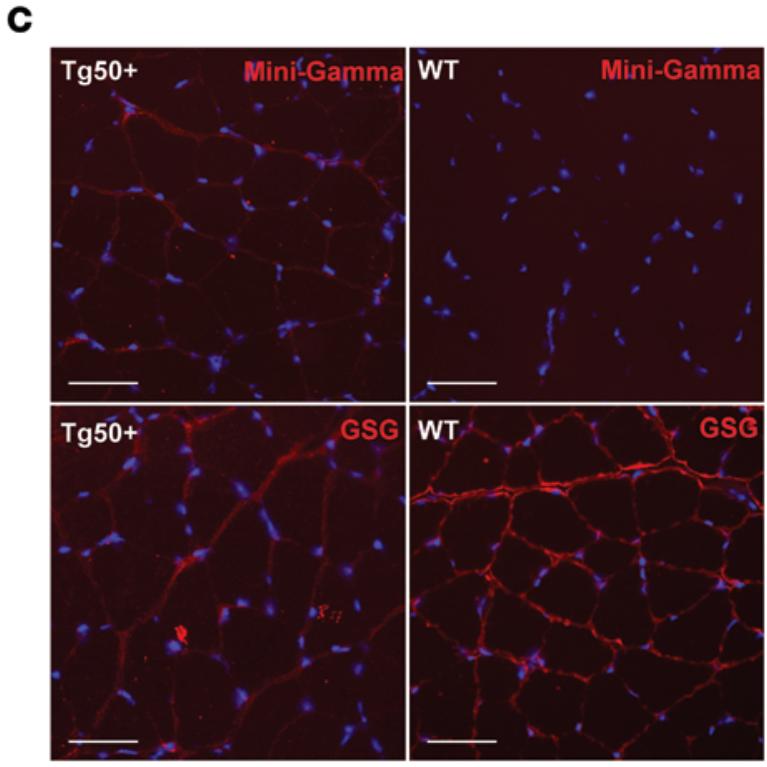

Figure 3. Mini-Gamma is incorporated into the sarcoglycan complex in vivo. (A) Transgenic mice expressing murine Mini-Gamma under the control of the human desmin (DES) promoter were generated. Two independent lines of Mini-Gamma were characterized; Tg50 had higher expression, while Tg84 had lower-level expression. (B) To assess sarcoglycan-complex formation, microsomal preparations were purified from transgenic mouse muscle. Membrane-associated microsomes were isolated. The sarcoglycan complex is known to enrich in the heavy microsomal fraction (HM), which contains the secretory system and plasma membrane (28). Similar to endogenous $\gamma$-sarcoglycan, Mini-Gamma was highly enriched in heavy microsomes isolated from both transgenic lines. (C) Mini-Gamma was found at the plasma membrane of skeletal muscle, as seen in cross sections from Tg50 ${ }^{+}$mouse muscle. Interestingly, endogenous $\gamma$-sarcoglycan was slightly diminished in $\mathrm{Tg}^{\circ} 0^{+}$animals compared with identically and simultaneously processed muscle sections from WT, suggesting competition for plasma membrane localization between Mini-Gamma and endogenous $\gamma$-sarcoglycan. Scale bars: $50 \mu m$. $\mathrm{H}$, heart muscle; S, skeletal muscle.

tein. Line Tg84 had lower-level expression, with Mini-Gamma expressed at $6 \%-9 \%$ of endogenous $\gamma$-sarcoglycan protein (Figure $3 \mathrm{~A})$. Muscle microsomal fractionation was used to monitor the expression of Mini-Gamma in muscle by separating fractions of crude total muscle lysates into cytoplasmic fraction, light microsomal, and heavy microsomal fractions. Sarcolemma, endoplasmic reticulum, and Golgi-associated membrane proteins are enriched in the heavy microsomal fraction. In WT animals, sarcoglycan proteins and other membrane-bound DGC components are mainly found in the muscle heavy microsomal fraction (28). Similar to the endogenous $\gamma$-sarcoglycan, Mini-Gamma protein was highly enriched in heavy microsomes from both transgenic lines indicating its normal trafficking in muscle (Figure 3B, HM lanes). Low amounts of Mini-Gamma were detected in the other membrane fractions, possibly consistent with competition between Mini-Gamma and full-length $\gamma$-sarcoglycan protein. To document intracellular localization, immunofluorescence microscopy was used to demonstrate the sarcolemmal pattern of Mini-Gamma protein in skeletal muscle (Figure 3C). Interestingly, endogenous $\gamma$-sarcoglycan was slightly diminished in Mini-Gamma transgenic animals compared with identically and simultaneously processed muscle sections from WT animals, suggesting competition for plasma membrane localization between Mini-Gamma and the endogenous $\gamma$-sarcoglycan protein (Figure 3C).

Mini-Gamma ameliorates skeletal muscle defects in Sgcg mice. Proper assembly of sarcoglycan complex is essential for its translocation to the plasma membrane in the muscle cells. In the absence of $\gamma$-sarcoglycan, sarcolemma targeting of $\beta$ - and $\delta$-sarcoglycan is impaired, reducing $\beta$ - and $\delta$-sarcoglycan content in the heavy microsomal fraction (21). Tg50 mice were crossed with $S g c g$-null animals to assess the capacity of Mini-Gamma to rescue the absence of $S g c g$. In $S g c g, T g 50$ animals, $\beta$ - and $\delta$-sarcoglycan protein levels were increased in the heavy microsomal fraction compared with those from Sgcg-null animals (Figure 4A, HM lanes). To test the interaction between Mini-Gamma and the other sarcoglycans in vivo, co-IP was performed from the heavy microsomal fraction. Mini-Gamma was co-IP, along with $\beta$-sarcoglycan (Figure 4B). The epitope tag antibody to Mini-Gamma also resulted in co-IP of $\beta$-sarcoglycan (Figure $4 \mathrm{~B}$ ). The presence of Mini-Gamma resulted in improved sarcolemma localization of $\beta$-sarcoglycan (Figure 4C), consistent with the microsomal membrane fractionation data in Figure 4A.

In many models of muscular dystrophy, the diaphragm muscle is one of the most severely diseased muscles, and the diaphragm muscle in $S g c g$-null mice is markedly thickened and has an increase in centrally nucleated myofibers (29). In $\mathrm{Sgcg}, \mathrm{Tg} 50$ mice, the thickness of the diaphragm muscle was reduced to normal size (Figure 4, $\mathrm{D}$ and $\mathrm{E}$ ). The percentage of centrally nucleated fibers was reduced in diaphragm muscle from $\mathrm{Sgcg}, \mathrm{Tg} 5 \mathrm{O}$ mice compared with $\mathrm{Sgcg}$-null mice (Figure $4 \mathrm{~F}$ ), consistent with an improved phenotype from the presence of Mini-Gamma. Evans Blue dye uptake was evaluated in skeletal muscles from $S g c g, T g 50$ and $S g c g$-null mice as a measure of sarcolemmal fragility and leak. By gross inspection, reduced uptake of dye was seen in $S g c g, T g 50$ compared with $S g c g$-null mus- 
A



C

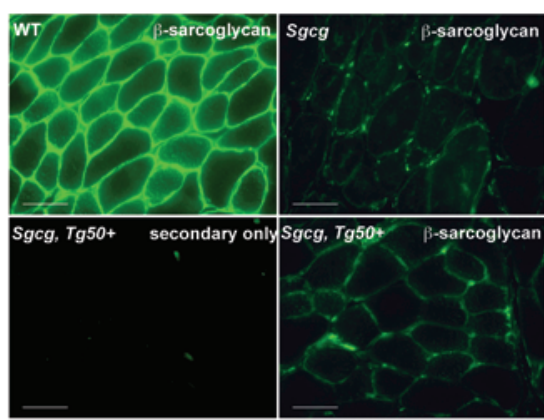

D
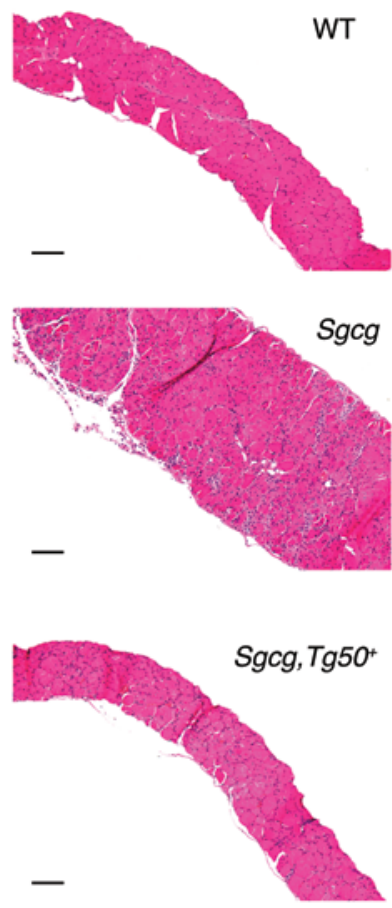

E
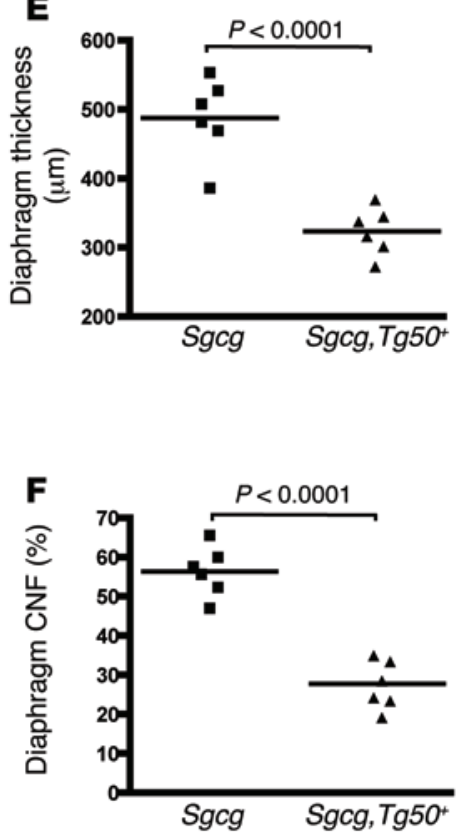

Figure 4. Mini-Gamma improves sarcoglycan trafficking and Sgcg mice skeletal muscle pathology. (A) In the absence of $\gamma$-sarcoglycan, $\beta$ - and $\delta$-sarcoglycan content in the heavy microsomal fraction is reduced due to impaired sarcolemma targeting, consistent with previous reports (21). In Sgcg,Tg50 mice, $\beta$ - and $\delta$-sarcoglycan protein levels were increased in the heavy microsomal fraction compared with those from Sgcg muscle without the Mini-Gamma transgene. (B) Co-IP from the heavy microsomal fraction was performed to test the interaction between Mini-Gamma and other sarcoglycans in vivo. Mini-Gamma was precipitated using an antibody against $\beta$-sarcoglycan ( $\alpha$-BSC). The Xpress tag antibody to Mini-Camma ( $\alpha$-MG) also resulted in precipitation of $\beta$-sarcoglycan. (C) Mini-Gamma expression improves $\beta$-sarcoglycan targeting to the sarcolemma. High-magnification imaging shows improved $\beta$-sarcoglycan localization in the presence of Mini-Gamma. Scale bars: $50 \mu \mathrm{m}$. (D) Mini-Gamma improves diaphragm muscle pathology in Sgcg mice. The diaphragm muscle is severely affected by the dystrophic process in Sgcg mice, as it is in other mouse models of muscular dystrophy, and this is seen as marked thickening, referred to as pseudohypertrophy (29). Scale bar: $100 \mu \mathrm{m}$. (E) In Sgcg, $T g 50$ mice, the thickness of the diaphragm muscle was reduced. (F) Centrally nucleated fibers (CNF), another feature of dystrophic muscle, are increased in Sgcg mice, reflecting increased pathological regeneration. The percentage of centrally nucleated fibers was reduced in diaphragm muscle from Sgcg,Tg50 mice compared with Sgcg mice, consistent with reduced degeneration and therefore a decreased need for regeneration. Student's $t$ test was used to compare results between 2 groups.

cle, and this was seen in diaphragm and abdominal muscles (Figure 5, A and B). Microscopically, dye uptake was reduced in quadriceps muscle (Figure $5 \mathrm{C}$ ). The percent of the diaphragm muscle opacified by dye uptake was reduced in $S g c g, T g 50$ compared with Sgcg-null muscle (Figure 5D). As a parallel measure of sarcolemmal leak, we also found that serum creatine kinase (CK) was reduced in $S g c g, T g 50$ compared with $S g c g$-null muscle (Figure 5E).

Mini-Gamma reduces fibrosis and improves function of $\mathrm{Sgcg}$ hearts. Because Mini-Gamma transgenic mice also expressed protein in cardiac muscle, we examined its effect on heart function. Protein expressed from the Mini-Gamma transgene was detected at the sarcolemma of cardiomyocytes from $\mathrm{Sgcg}, \mathrm{Tg} 50$ mice (Figure $6 \mathrm{~A}) . S g c g$ mice develop cardiac dysfunction and fibrosis as they age (29). Fibrosis, as monitored by hydroxyproline (HOP) content, was reduced in the hearts of $\mathrm{Sgcg}, \mathrm{Tg} 84$ mice compared with $\mathrm{Sgcg \text {-null }}$ mice (Figure 6B). In addition, $S g c g, T g 84$ animals had improved fractional shortening compared with $S g c g$-null animals (Figure 6C). This observation was made in male mice, since female $\mathrm{Sgcg}$ mice have greater variability in physiological measurements, and these findings were significant in the lower expressing MiniGamma transgenic line (Tg84). Tg50 expresses protein at levels higher than endogenous $\gamma$-sarcoglycan, and it has previously been shown that overexpression of $\gamma$-sarcoglycan is sufficient to induce findings of muscular dystrophy (30). These data demonstrate that Mini-Gamma improved cardiac dysfunction caused by loss of fulllength $\gamma$-sarcoglycan.

Exon skipping in LGMD $2 \mathrm{C}$ myogenic cells. Fibroblasts were obtained from an individual with LGMD 2C. This individual carried a large deletion of 1.4 MB spanning 7 genes, including $S G C G$ encoding $\gamma$-sarcoglycan on 1 allele. The other allele was deleted for 14,000 bp that encompassed only exon 6 of SGCG (Figure $7 \mathrm{~A})$, leading to a premature stop codon and disrupting the reading frame. The individual was diagnosed with LGMD $2 \mathrm{C}$ with progressive muscle weakness and elevated serum CK, which began in early childhood. A muscle biopsy confirmed absent $\gamma$-sarcoglycan protein and reduction of the other sarcoglycan proteins (data not shown). Fibroblasts were obtained and induced into a myogenic lineage using a tamoxifen-inducible (Tam-inducible) MyoD, following similar methods used to examine DMD cells (refs. 31, 32, and Supplemental Figure 5). After induction, MyoD-reprogrammed fibroblasts entered into the myogenic lineage seen as desmin expression, along with the appearance of elongated myo- 
A
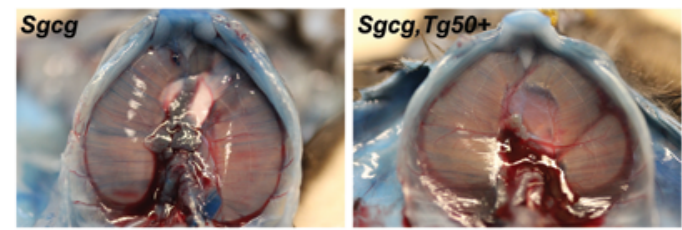

B
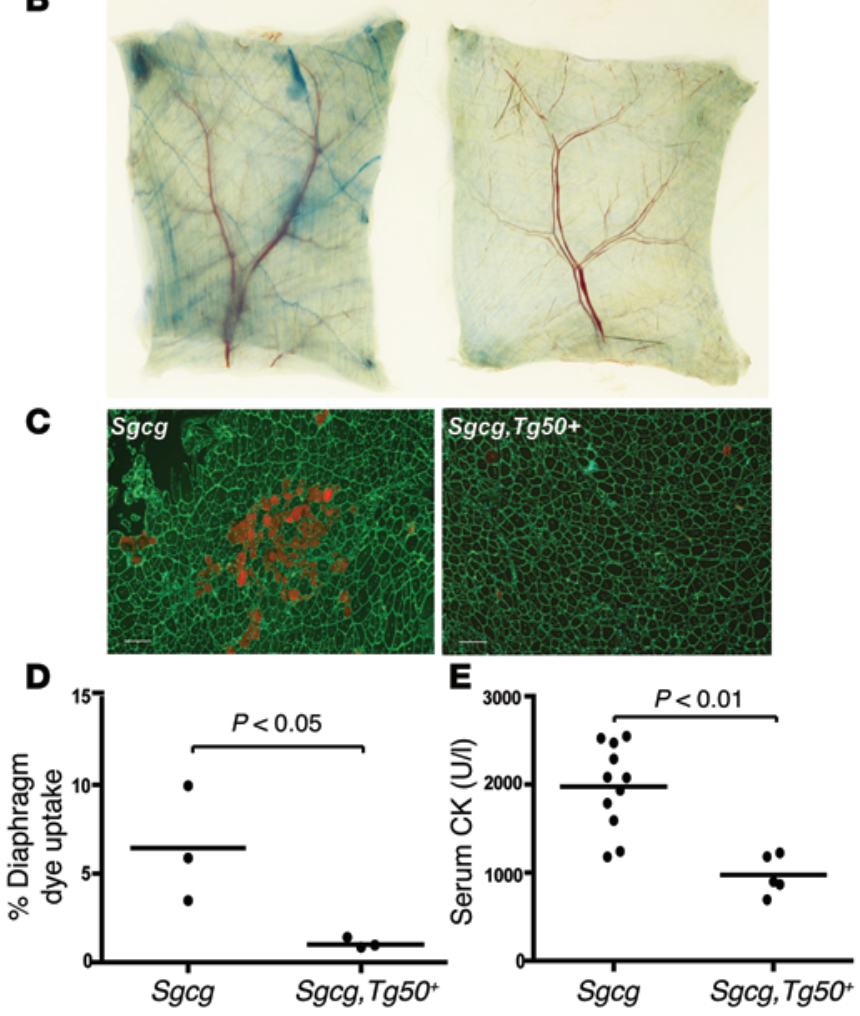

tube-like structures (Figure 7B). LGMD 2C fibroblasts entered into the myogenic lineage similar to WT control cells. SGCG RNA expression was detected in MyoD-reprogrammed fibroblasts (Figure $7 \mathrm{C}$ ) from both the control and the LGMD $2 \mathrm{C}$ patient (ex6del). The degree of SGCG RNA expression was qualitatively less in ex6del, consistent with the presence of a single functional SGCG allele and/or nonsense-mediated decay.

AONs using 2'-O-methyl phosphorothioate (2OMePS) chemistry were targeted to intraexonic regions in $S G C G$ exons 4,5 , and 7 , in accordance with previously described principles for antisense design (33). Transfection of reprogrammed ex6del cells with single 2OMePS AONs demonstrated dose-dependent skipping of individual exons 5 and 7, with less robust single skipping of exon 4 (Supplemental Figure 6). Interestingly, there was evidence for endogenous skipping of exon 7 in the absence of AONs to exon 7 (Supplemental Figure 6). To generate the multiexon-skipped and reading frame-corrected ex6del transcript, reprogrammed cells were treated with a cocktail of AONs targeting exons 4,5 , and 7 $(100 \mathrm{nM} / \mathrm{AON}, 300 \mathrm{nM}$ total). Analysis of PCR-amplified transcripts 3 days after treatment demonstrated the generation of an internally truncated transcript with the desired reading frame correction of ex6del SGCG (Figure 7D), in addition to the intermediate skipped products. The 3-exon-skipped product was confirmed by sequencing (Supplemental Figure 7). The cell culture system used here exhibits reduced survival with persistent high-
Figure 5. Reduction of sarcolemmal leak with Mini-Gamma expression in Sgcg-null mice. Evans blue dye was injected into Sgcg-null mice to monitor sarcolemmal fragility and subsequently leak. (A and $\mathbf{B}$ ) Gross images are shown from the diaphragm muscle (top row) and abdominal muscle (middle row) demonstrating dye uptake (blue). (c) Immunofluorescence images of quadriceps muscle sections with dystrophin staining (green) and dye uptake marked as red. Scale bars: $200 \mu \mathrm{m}$. The reduction of dye uptake was observed for each muscle by the presence of the Mini-Gamma transgene. (D) The percentage of the diaphragm muscle that was opacified by dye was measured and was reduced in $\mathrm{Sgcg}, \mathrm{Tg} 50$ muscle compared with Sgcg-null muscle. (E) Serum CK was reduced in Sgcg,Tg50 compared with Sgcg-null mice. Reduction of CK is another reflection of improved muscle-membrane stability.

level MyoD and AON treatment, thus limiting the ability to measure outcomes after more than a few days and limiting detection of Mini-Gamma protein. Collectively, these data demonstrate the potential of correcting SGCG frameshift mutations with a multiexon skipping strategy.

\section{Discussion}

Gene therapy for muscular dystrophy has often relied on viral strategies for gene replacement. For DMD, this has been especially problematic due to the large size of the dystrophin gene and protein, along with the limited capacity of viral vectors. For LGMD $2 \mathrm{C}$, which is caused by SGCG mutations and results in the loss of much smaller $\gamma$-sarcoglycan protein, viral gene therapy was initiated (34). However, viral gene therapy for any muscle disease is challenged by the need to deliver the product to all muscle groups. Because of these hurdles, exon skipping is emerging as a genetic-correction strategy of clinical utility. The progress in clinical trials for DMD suggests that exon skipping could be applied to other disorders, including neuromuscular disorders. The premise for exon skipping for DMD derives from observations in BMD patients with in-frame deletions that preserved the functionally important amino- and carboxy-termini of dystrophin. The X-linked recessive nature of DMD, combined with the repetitive structure of the dystrophin rod domain, made DMD an ideal target for exon skipping. Although an attractive method for genetic correction, exon skipping in DMD typically yields a much lower level of dystrophin protein compared with endogenous dystrophin levels. This lower level of expression of what is essentially an abnormal protein likely explains the partial correction observed in clinical trials (35). For DMD, detection of the protein product produced from exon skipping has been challenging, raising questions of whether dystrophin protein production is a suitable biomarker (36).

Whether exon skipping is useful for other disorders requires documentation that internally truncated proteins are functional. Here, we show that Mini-Gamma protein is able to biochemically replace full-length $\gamma$-sarcoglycan in flies, in mouse heart and muscle, and in a heterologous cell expression system by forming a complex with the $\beta$ - and $\delta$-sarcoglycans and promoting translocation to the membrane. Furthermore, we showed evidence for functional improvement by Mini-Gamma in a Drosophila model of sarcoglycan deficiency where mobility and heart function were each improved. In the Sgcg-null mouse, we found that MiniGamma improved cardiac dysfunction. Of note, this model does not allow us to detect functional improvement using ex vivo mus- 
A

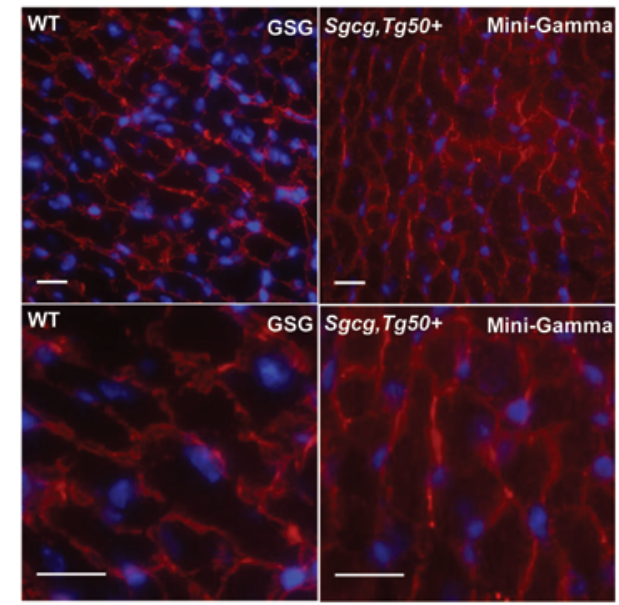



Figure 6. Mini-Gamma reduces fibrosis and improves function of Sgcg hearts. (A) Mini-Gamma protein was detected at the sarcolemma of cardiomyocytes from Sgcg,Tg50 mice, similar to that of the endogenous $\gamma$-sarcoglycan in WT animals. Scale bars: $20 \mu \mathrm{m}$. (B) Sgcg mice develop fibrosis and impaired cardiac function (29). Cardiac fibrosis was monitored by HOP content. In Sgcg,Tg84 mice, heart fibrosis was reduced compared with $\mathrm{Sgcg}$ mice. (C) Cardiac function was evaluated by echocardiography. Compared with Sgcg mice, Sgcg,Tg84 mice had improved fractional shortening. Sgcg,Tg50 trended toward improvement in fibrosis and fractional shortening, and this may be due to toxic overexpression of Mini-Gamma, as overexpression of $\gamma$-sarcoglycan in skeletal muscle can induce muscular dystrophy (30). Fibrosis and fractional shortening measurements shown here are of males only. Females showed the same trends but not significantly (data not shown). ${ }^{*} P<0.05$. Comparisons were made using one-way ANOVA.

cle mechanics. Sgcg-null skeletal muscle differs from dystrophin-deficient muscle in that dystrophin-deficient muscle displays contraction-induced damage while $S g c g$-null muscle does not have increased force deficit from eccentric contraction $(37,38)$. Dystrophin, with its multi-spectrin repeat nature, creates a mechanically strong link for the sarcolemma (39), and this is in contradistinction to $S g c g$-null muscle in which dystrophin is normally localized (38).

Although the strategy to generate Mini-Gamma removes half of $\gamma$-sarcoglycan, it retains the components integral to $\gamma$-sarcoglycan function. $\gamma$-Sarcoglycan is a type II transmembrane protein with a 37 aa intracellular amino-terminus, a 21 aa transmembrane domain, and a 233 aa extracellular domain. Exon 2 encodes the initiator methionine and the entire intracellular and transmembrane domains, and these domains remained intact in MiniGamma. Notably, the existing mouse model for LGMD 2C was deleted for exon 2 and is therefore not a suitable model for testing exon skipping (29). The intracellular amino-terminus of $\gamma$-sarcoglycan contains tyrosine phosphorylation consensus sequences; tyrosine phosphorylation is seen with cell attachment and contraction, and it is required for proper mechanosignaling (40-44). The intracellular domain has also been shown to interact directly with intermediate filament protein filamin- $\mathrm{C}$ and actin-associated protein archvillin $(43,45)$. The amino-terminal half of the extracellular domain is important for interacting with other sarcoglycans during complex assembly (46). The interaction of Mini-Gamma with the other sarcoglycans and its translocation to the plasma membrane indicate that the residual extracellular portion of $\gamma$-sarcoglycan was sufficient for membrane targeting. The carboxy-terminal extracellular region of $\gamma$-sarcoglycan contains an EGF-like cysteine-rich domain that is conserved among $\beta$-, $\delta$-, and $\gamma$-sarcoglycan. Notably, this region remained intact in MiniGamma (14, 16, 47). This cysteine-rich motif found at the distal carboxy-terminus of $\gamma$-, $\beta$-, and $\delta$-sarcoglycans has been shown to form intramolecular disulfide bridges and is required for plasma membrane targeting $(26,46,48)$. Missense mutations of these cysteines and small deletions in this region cause severe forms of human muscular dystrophy $(49,50)$.

The most common mutation in the $S G C G$ gene is a frameshiftin exon 6,521- $\Delta \mathrm{T}$ $(15,47)$. An exon-skipping strategy that includes exon 6 is expected to benefit not only patients carrying the 521- $\Delta \mathrm{T}$ mutation, which accounts for about half of all LGMD $2 \mathrm{C}$ patients, but also patients carrying missense, nonsense, or frame-shifting mutations spanning from exon 4-7. One concern for an exon-skipping strategy is that nonsense-mediated mRNA decay mechanisms may leave little or no mRNA transcript (51). However, we identified sufficient RNA produced from a single $S G C G$ allele, suggesting that this may not be a significant hurdle.

Like exon skipping for DMD, the presence of an internally truncated protein - likely at protein levels much lower than normal, as exon skipping remains an inefficient process - is anticipated to produce only partial rescue of phenotype. This study demonstrates that AON-mediated skipping is plausible, since the mRNA product can be detected. However, further optimization is required to demonstrate that Mini-Gamma protein is expressed in myogenic-like cell lines from human patients. This may require testing in myoblasts isolated from human muscle, since the MyoDinduced system produces myogenic cells of limited lifespan. This partial rescue is consistent with what is seen in the milder BMD patients and still represents a significant improvement in phenotype. Improved chemistries for antisense approaches should lead to more efficient skipping and higher levels of appropriate RNA templates. Permanent genetic therapy may one day be achievable through gene editing.

\section{Methods}

Plasmids. Murine Sgcg and Mini-Gamma were ligated into pUAST vector (17), and an Xpress epitope tag was added. The pUAST-MiniGamma was digested and inserted into pcDNA3.0 vector at EcoR1 and Xho1 sites to generate plasmids for expression in HEK293T cells. Mouse Sgcb (MR204617) and mouse Sgcd (MR221060) cDNA ORF clones were purchased from OriGene. Both vectors contain CMV promoters and Myc-DDK tags at the $\mathrm{C}$-terminus of the respective sarcoglycan protein.

Drosophila strains. pUAST-Sgcg and pUAST-Mini-Gamma plasmids were integrated using P element insertion (Rainbow Transgenic Flies Inc.). Founder males were mated to $y[1] w[1118]$ (yw) females, 
A

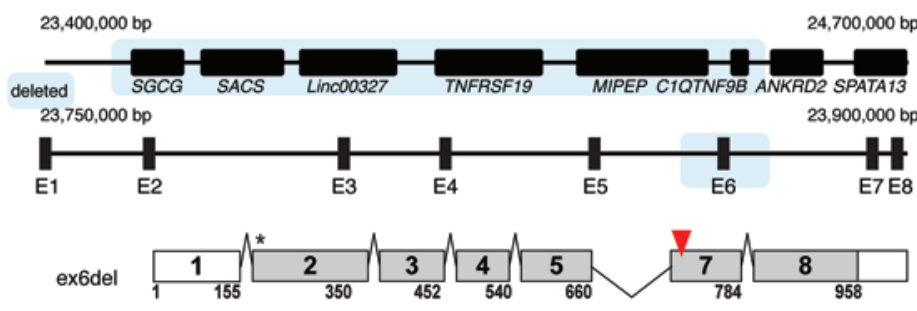

B MyoD treated Nuclei


C

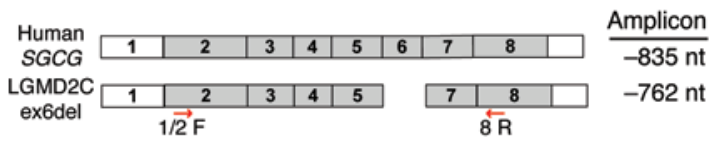

SGCG MRNA (-Tam)

GCG mRNA (+Tam)

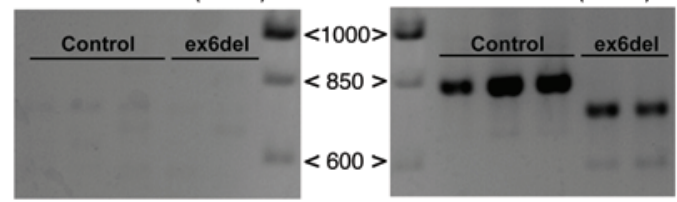

D

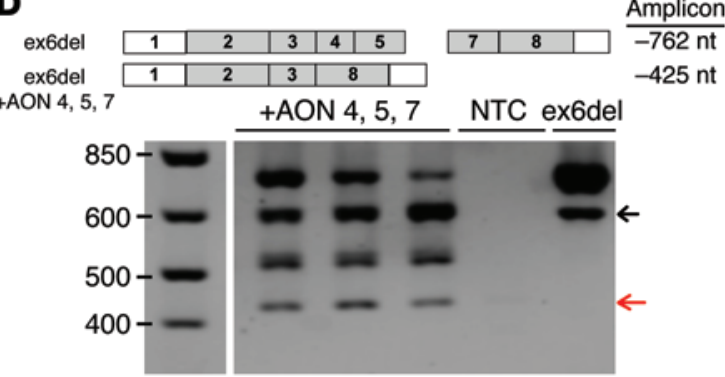

Figure 7. AON-mediated reading frame correction in human SGCG mutant cells. (A) Shown are the 2 different alleles from an individual with LGMD $2 C$ affecting the SGCG locus, a 1.4 MB deletion encompassing multiple genes (blue shaded area, top) and a smaller deletion encompassing SGCG exon 6 in its entirety (blue shaded area, middle schematic). Numbers refer to genome position in hg19. The exon organization for exon 6 deletion (ex6del) mutant SGCG transcripts is shown in the lower schematic. Asterisk represents transcription start site. Red arrowhead indicates premature stop codon. (B) Skin fibroblasts from control and ex6del were obtained and reprogrammed to the myogenic lineage using a tamoxifen-inducible MyoD (31, 32). Desmin expression (red) and multinucleated myotube formation indicated myogenic reprogramming after $40 \mathrm{H}$-tamoxifen exposure $(5 \mu \mathrm{M}, 48$ hours) and culture in differentiation media. Nuclei were marked with Hoechst 3342 (blue). Scale bars: $10 \mu \mathrm{M}$. (C) RT-PCR demonstrated SGCC transcripts from control and ex6del mutant in reprogrammed (right) fibroblasts after differentiation ( $5 \mu \mathrm{M} 4 \mathrm{OH}$-tamoxifen, 48 hours; 12-day differentiation). (D) MyoD-reprogrammed fibroblasts were treated with AONs targeting exons 4, 5, and $7(100 \mathrm{nM} / \mathrm{AON}, 300 \mathrm{nM}$ total). RT-PCR demonstrated the expected skipped products, including the smallest product representing exons 2, 3, and 8 and deleted for exons 4, 5, and 7 (red arrow). Results from 3 independent replicates are shown for AON treatment. NTC, notemplate control. Black arrow indicates the single exon skipping of exon 7 in the ex6del SGCG transcript, which occurs in the absence of AON treatment.

and their progeny were screened for the presence of $\mathrm{w}[+\mathrm{mC}]$. The TinC44-Gal4 strain was a gift from Manfred Frasch (University of Erlangen-Nuremberg, Nuremberg, Germany) (52). Mef2-Gal4 and MHC-Gal4 were gifts from Ron Dubreuil (University of Illinois at Chicago, Chicago, Illinois, USA) $(53,54)$. The $S g c d^{840}$ strain was previously described (18). The Drosophila strain yw was used as the WT control in all studies (Bloomington Drosophila Stock Center). $S g c d^{840}$ strain and all transgenic fly strains were backcrossed with the $y w$ strain for 6 generations to allow homogenization across the whole genome.

Drosophila activity assay. The MB5 MultiBeam Activity Monitor (TriKinetics) was used to quantify fly basal activity, and all activity assays were performed on flies that had been aged to 20 days after eclosion.

OCT. OCT was performed as previously described $(24,55)$.

Antibodies. MGSG protein was detected with a rabbit polyclonal anti- $\gamma$-sarcoglycan antibody (47). A rabbit polyclonal antibody was raised to the Xpress epitope (Pocono Rabbit Farm and Laboratory Inc.) and affinity-purified. The rabbit polyclonal anti-SGCG antibody NBP1-90298 was used (Novus Biologicals). $\beta$-Sarcoglycan was detected with NCL-b-SARC (Leica Microsystems) and rabbit polyclonal anti-SGCB antibody NPB1-19782 (Novus Biologicals). $\delta$-Sarcoglycan was detected with a polyclonal antibody (21). Dystrophin was detected with anti-dystrophin antibody RB-9024-P (Thermo Electron Corp.) Secondary antibodies were Alexa Fluor 488 goat anti-rabbit and Alexa Fluor 594 goat anti-rabbit 594 (Invitrogen). Horseradish peroxidase-conjugated goat anti-rabbit and goat anti-mouse were used in immunoblotting (Jackson ImmunoResearch Laboratories Inc.).

Microsome preparation. Membrane-bound proteins were isolated following previously specified protocol (28), with modifications.

Co-IP. Co-IP was performed according to published protocols (21) with modifications.

HOP assay and echocardiography. HOP content was determined as previously described (56). Echocardiography was performed as previously described (57).

In vitro human cell culture and AON transfection. Primary fibroblasts were derived from a skin biopsy obtained from an LGMD 2C patient. Primary fibroblasts from a healthy control subject (CRL-2565) were obtained from the ATCC. LGMD 2C (ex6del, e6d) and control cells were transduced with an inducible MyoD lentiviral construct (iMyoD), previously described $(31,32)$. After $4 \mathrm{OH}$-tamoxifen induction and culture in differentiation media ( $5 \mu \mathrm{M} / 48$ hours; $10-12$ day differentiation), fibroblasts were reprogrammed to express myogenic markers, including the SGCG transcript, and formed multinucleated myotubes. Exon 
skipping with AONs utilized 2OMePS chemistry. AONs were designed in accordance with previously described guidelines and synthesized by Integrated DNA Technologies(33). Sequences are defined in Supplemental Table 1. 2OMePS were transfected into cells on differentiation day 9 (6 hours, $100 \mathrm{nM}$ per AON) in serum-free media. On day 12, total RNA was isolated from cells, reverse transcribed, and evaluated for fulllength and internally truncated SGCG expression via PCR and gel electrophoresis. Additional information regarding the experimental procedures can be found in the online Supplemental Material.

Statistics. Prism (GraphPad Software) was used for data analysis. Student's $t$ test was used to compare results between 2 groups. $P<0.05$ was considered significant.

Study approval. Written and informed consent was obtained in accordance with the University of Chicago's Institutional Review
Board. All work was conducted under the University of Chicago and Northwestern's Institutional Review Boards.

\section{Acknowledgments}

We thank the Kurt + Peter Foundation for facilitating these studies, and the patient and families for participation. We thank Jeff Chamberlain for providing the inducible MyoD construct. This work was supported by NIH R01HL61322 and U54AR052646 (to E.M. McNally), and NIH R01HL116581 (to M.J. Wolf).

Address correspondence to: Elizabeth McNally, Center for Genetic Medicine, 303 E. Superior St., Northwestern University, Chicago, Illinois 60302, USA. Phone: 312.503.5600; E-mail: elizabeth.mcnally@northwestern.edu.
1. Durbeej M, Campbell KP. Muscular dystrophies involving the dystrophin-glycoprotein complex: an overview of current mouse models. Curr Opin Genet Dev. 2002;12(3):349-361.

2. Ervasti JM. Dystrophin, its interactions with other proteins, and implications for muscular dystrophy. Biochim Biophys Acta. 2007;1772(2):108-117.

3. Rahimov F, Kunkel LM. The cell biology of disease: cellular and molecular mechanisms underlying muscular dystrophy. J Cell Biol. 2013;201(4):499-510.

4. Ervasti JM, Campbell KP. A role for the dystrophin-glycoprotein complex as a transmembrane linker between laminin and actin. JCell Biol. 1993;122(4):809-823.

5. Cohn RD, Campbell KP. Molecular basis of muscular dystrophies. Muscle Nerve. 2000;23(10):1456-1471.

6. Kinali M, et al. Local restoration of dystrophin expression with the morpholino oligomer AVI-4658 in Duchenne muscular dystrophy: a single-blind, placebo-controlled, dose-escalation, proof-of-concept study. Lancet Neurol. 2009;8(10):918-928.

7. Cirak S, et al. Exon skipping and dystrophin restoration in patients with Duchenne muscular dystrophy after systemic phosphorodiamidate morpholino oligomer treatment: an openlabel, phase 2, dose-escalation study. Lancet. 2011;378(9791):595-605.

8. van Deutekom JC, et al. Local dystrophin restoration with antisense oligonucleotide PRO051. N Engl JMed. 2007;357(26):2677-2686.

9. Goemans NM, et al. Systemic administration of PRO051 in Duchenne's muscular dystrophy. N Engl JMed. 2011;364(16):1513-1522.

10. Lu QL, Cirak S, Partridge T. What can we learn from clinical trials of exon skipping for DMD? Mol Ther Nucleic Acids. 2014;3:e152.

11. Ervasti JM, Campbell KP. Membrane organization of the dystrophin-glycoprotein complex. Cell. 1991;66(6):1121-1131.

12. Ozawa E, Mizuno Y, Hagiwara Y, Sasaoka T, Yoshida M. Molecular and cell biology of the sarcoglycan complex. Muscle Nerve. 2005;32(5):563-576.

13. Roberds SL, et al. Missense mutations in the adhalin gene linked to autosomal recessive muscular dystrophy. Cell. 1994;78(4):625-633.

14. Bonnemann CG, et al. $\beta$-Sarcoglycan (A3b) mutations cause autosomal recessive muscular dystrophy with loss of the sarcoglycan complex. Nat Genet. 1995;11(3):266-273.

15. Noguchi S, et al. Mutations in the dystrophin-associated protein gamma-sarcoglycan in chromosome 13 muscular dystrophy. Science. 1995;270(5237):819-822.

16. Nigro V, et al. Autosomal recessive limb-girdle muscular dystrophy, LGMD2F, is caused by a mutation in the delta-sarcoglycan gene. Nat Genet. 1996;14(2):195-198.

17. Brand AH, Perrimon N. Targeted gene expression as a means of altering cell fates and generating dominant phenotypes. Development. 1993;118(2):401-415.

18. Allikian MJ, et al. Reduced life span with heart and muscle dysfunction in Drosophila sarcoglycan mutants. Hum Mol Genet. 2007;16(23):2933-2943.

19. Vainzof M, et al. The sarcoglycan complex in the six autosomal recessive limb-girdle muscular dystrophies. Hum Mol Genet. 1996;5(12):1963-1969.

20. Mizuno Y, et al. Selective defect of sarcoglycan complex in severe childhood autosomal recessive muscular dystrophy muscle. Biochem Biophys Res Commun. 1994;203(2):979-983.

21. Hack AA, et al. Differential requirement for individual sarcoglycans and dystrophin in the assembly and function of the dystrophin-glycoprotein complex. J Cell Sci. 2000;113(pt 14):2535-2544.

22. Durbeej M, et al. Disruption of the $\beta$-sarcoglycan gene reveals pathogenetic complexity of limb-girdle muscular dystrophy type 2E. Mol Cell. 2000;5(1):141-151.

23. Duclos F, et al. Progressive muscular dystrophy in alpha-sarcoglycan-deficient mice. J Cell Biol. 1998;142(6):1461-1471.

24. Wolf MJ, Amrein H, Izatt JA, Choma MA, Reedy MC, Rockman HA. Drosophila as a model for the identification of genes causing adult human heart disease. Proc Natl Acad Sci US A. 2006;103(5):1394-1399.

25. Noguchi S, Wakabayashi E, Imamura M, Yoshida M, Ozawa E. Formation of sarcoglycan complex with differentiation in cultured myocytes. Eur J Biochem. 2000;267(3):640-648.

26. Shi W, Chen Z, Schottenfeld J, Stahl RC, Kunkel LM, Chan YM. Specific assembly pathway of sarcoglycans is dependent on beta- and delta-sarcoglycan. Muscle Nerve. 2004;29(3):409-419.
27. Pacak CA, Sakai Y, Thattaliyath BD, Mah CS, Byrne BJ. Tissue specific promoters improve specificity of AAV9 mediated transgene expression following intra-vascular gene delivery in neonatal mice. Genet Vaccines Ther. 2008;6:13.

28. Ohlendieck K, Campbell KP. Dystrophin-associated proteins are greatly reduced in skeletal muscle from mdx mice. JCell Biol. 1991;115(6):1685-1694.

29. Hack AA, et al. $\gamma$-Sarcoglycan deficiency leads to muscle membrane defects and apoptosis independent of dystrophin. JCell Biol. 1998;142(5):1279-1287.

30. Zhu X, Hadhazy M, Groh ME, Wheeler MT, Wollmann R, McNally EM. Overexpression of $\gamma$-sarcoglycan induces severe muscular dystrophy. Implications for the regulation of Sarcoglycan assembly. J Biol Chem. 2001;276(24):21785-21790.

31. Kimura E, et al. Cell-lineage regulated myogenesis for dystrophin replacement: a novel therapeutic approach for treatment of muscular dystrophy. Hum Mol Genet. 2008;17(16):2507-2517.

32. Kendall GC, et al. Dantrolene enhances antisense-mediated exon skipping in human and mouse models of Duchenne muscular dystrophy. Sci Transl Med. 2012;4(164):164ra0.

33. Aartsma-Rus A. Overview on AON design. Methods Mol Biol. 2012;867:117-129.

34. Herson S, et al. A phase I trial of adeno-associated virus serotype $1-\gamma$-sarcoglycan gene therapy for limb girdle muscular dystrophy type 2C. Brain. 2012;135(pt 2):483-492.

35. Voit $\mathrm{T}$, et al. Safety and efficacy of drisapersen for the treatment of Duchenne muscular dystrophy (DEMAND II): an exploratory, randomised, placebo-controlled phase 2 study. Lancet Neurol. 2014;13(10):987-996.

36. Wilton SD, Fletcher S, Flanigan KM. Dystrophin as a therapeutic biomarker: are we ignoring data from the past? Neuromuscul Disord. 2014;24(6):463-466.

37. Petrof BJ, Shrager JB, Stedman HH, Kelly AM, Sweeney HL. Dystrophin protects the sarcolemma from stresses developed during muscle contraction. Proc Natl Acad Sci US A 1993;90(8):3710-3714.

38. Hack AA, Cordier L, Shoturma DI, Lam MY, Sweeney HL, McNally EM. Muscle degeneration without mechanical injury in sarcoglycan deficiency. Proc Natl Acad Sci U S A. 
1999;96(19):10723-10728.

39. Rybakova IN, Patel JR, Ervasti JM. The dystrophin complex forms a mechanically strong link between the sarcolemma and costameric actin. JCell Biol. 2000;150(5):1209-1214.

40. Yoshida T, Pan Y, Hanada H, Iwata Y, Shigekawa M. Bidirectional signaling between sarcoglycans and the integrin adhesion system in cultured L6 myocytes. J Biol Chem. 1998;273(3):1583-1590.

41. Barton ER. Impact of sarcoglycan complex on mechanical signal transduction in murine skeletal muscle. Am J Physiol Cell Physiol. 2006;290(2):C411-C419.

42. Barton ER. Restoration of gamma-sarcoglycan localization and mechanical signal transduction are independent in murine skeletal muscle. J Biol Chem. 2010;285(22):17263-17270.

43. Spinazzola JM, Smith TC, Liu M, Luna EJ, Barton ER. $\gamma$-Sarcoglycan is required for the response of archvillin to mechanical stimulation in skeletal muscle. Hum Mol Genet. 2015;24(9):2470-2481.

44. Moorwood C, Philippou A, Spinazzola J, Keyser B, Macarak EJ, Barton ER. Absence of $\gamma$-sarcoglycan alters the response of p70s6 kinase to mechanical perturbation in murine skeletal muscle. Skelet
Muscle. 2014;4:13.

45. Thompson TG, et al. Filamin 2 (FLN2): a muscle-specific sarcoglycan interacting protein. J Cell Biol.2000;148(1):115-126.

46. Chen J, et al. Identification of functional domains in sarcoglycans essential for their interaction and plasma membrane targeting. Exp Cell Res. 2006;312(9):1610-1625.

47. McNally EM, et al. Mild and severe muscular dystrophy caused by a single $\gamma$-sarcoglycan mutation. Am J Hum Genet. 1996;59(5):1040-1047.

48. Chan YM, Bonnemann CG, Lidov HG, Kunkel LM. Molecular organization of sarcoglycan complex in mouse myotubes in culture. J Cell Biol. 1998;143(7):2033-2044.

49. Piccolo F, et al. A founder mutation in the gammasarcoglycan gene of gypsies possibly predating their migration out of India. Hum Mol Genet. 1996;5(12):2019-2022.

50. McNally EM, et al. Mutations that disrupt the carboxyl-terminus of $\gamma$-sarcoglycan cause muscular dystrophy. Hum Mol Genet. 1996;5(11):1841-1847.

51. Baker KE, Parker R. Nonsense-mediated mRNA decay: terminating erroneous gene expression. Curr Opin Cell Biol. 2004;16(3):293-299.
52. Lo PC, Frasch M. A role for the COUP-TFrelated gene seven-up in the diversification of cardioblast identities in the dorsal vessel of Drosophila. Mech Dev. 2001;104(1-2):49-60.

53. Ranganayakulu G, Schulz RA, Olson EN. Wingless signaling induces nautilus expression in the ventral mesoderm of the Drosophila embryo. Dev Biol. 1996;176(1):143-148.

54. Schuster CM, Davis GW, Fetter RD, Goodman CS. Genetic dissection of structural and functional components of synaptic plasticity. I. Fasciclin II controls synaptic stabilization and growth. Neuron. 1996;17(4):641-654.

55. Wolf MJ, Rockman HA. Drosophila melanogaster as a model system for genetics of postnatal cardiac function. Drug Discov Today Dis Models. 2008;5(3):117-123.

56. Heydemann A, Huber JM, Demonbreun A, Hadhazy M, McNally EM. Genetic background influences muscular dystrophy. Neuromuscul Disord. 2005;15(9-10):601-609.

57. Goldstein JA, et al. Excess SMAD signaling contributes to heart and muscle dysfunction in muscular dystrophy. Hum Mol Genet. 2014;23(25):6722-6731. 